\title{
Neck Swelling in A Child: Now You See it, Now You Don't?
}

\author{
Ikram Hakim ${ }^{a}$, Goh Bee See ${ }^{a}$, Hamzaini Abd Hamid \\ ${ }^{a}$ Department of Otorhinolaryngology and Head and Neck Surgery, Universiti Kebangsaan Malaysia Medical \\ Center (UKMMC), Kuala Lumpur, Malaysia. \\ ${ }^{b}$ Department of Radiology, Universiti Kebangsaan Malaysia Medical Center (UKMMC), Kuala Lumpur, \\ Malaysia.
}

\section{ABSTRACT}

Jugular Ectasia is a rare benign swelling due to dilatation of jugular vein, which can occur in the internal, external or an anterior jugular vein. It is characterized by painless, soft, compressible unilateral swelling appeared on Valsalva maneuver. A 3-year-old boy presented with 2 months history of prominent mass over the right side of the neck on Valsalva maneuver is subjected to Doppler ultrasonography (USG) of the neck. Doppler Ultrasonography (USG) of the neck revealed prominent right jugular dilatation during Valsalva without any focal lesion with the normal caliber of the left internal jugular vein. Jugular ectasia should be included in the differentials of a benign neck swelling in children despite infrequently encountered. Dilated jugular vein on ultrasound Doppler on Valsalva maneuver is pathognomic of jugular ectasia. Early diagnosis with serial follow up can reduce parent's anxiety and will reduce complications.

Keywords: Venous Phlebectasia, Internal jugular vein, Ectasia, Doppler Ultrasonography, Paediatric neck mass

\section{INTRODUCTION}

Venous ectasia or phlebectasia is a condition of venous dilatation. It is a challenging diagnosis due to limited resources among the medical fraternity. Jugular vein ectasia commonly presented as soft, unilateral compressible swelling on the neck, which will appear more obvious during increase intrathoracic pressure, such as crying, straining, sneezing or coughing and Valsalva maneuver. ${ }^{1}$ It is often mistaken for laryngocele, cystic hygroma, branchial cyst, AV malformation and superior mediastinal mass. ${ }^{2}$ Interestingly, jugular vein ectasia is rarely reported in the literature and the incidence worldwide is undetermined.

USG combined with Doppler flow imaging is a gold standard to diagnose jugular ectasia. ${ }^{3}$ Due to its benign nature, many considered conservative follow

Corresponding author:

Dr Goh Bee See

Dept. of Otorhinolaryngology and Head and Neck Surgery

Level 9, Clinical Block, Hospital Canselor Tuanku Mukhriz,

Universiti Kebangsaan Malaysia Medical Center, Jalan Yaacob Latiff, Bandar Tun Razak, 56000 Cheras, Kuala Lumpur, Malaysia.

Tel No: +6039769 2434

Email : irenegbs@yahoo.com up is recommended for this condition, although surgical resection has been reported for the symptomatic patient.

\section{CASE REPORT}

A 3-year-old boy premorbid healthy presented to the Otorhinolaryngology clinic in Universiti Kebangsaan Malaysia Medical Center, Cheras, with swelling over the right side of the neck for 2 months. The mother noticed that her child has an intermittent right side of the neck swelling. The swelling became more prominent, especially during crying and laughing. The child did not have any other active complaint, such as pain, discharge or tenderness over the site. He has no other contributory compressive symptoms such as noisy breathing, hoarseness or dysphagia. The mother denied any history of trauma prior illness or injury at the site. His physical examination was normal except for a soft, compressible swelling over the right side of the neck at level IV during the Valsalva maneuver. There was no thrill palpable or bruit auscultated on the mass. Flexible video endoscopy revealed normal larynx without medialisation of the lateral pharyngeal wall. Bilateral vocal cords were normal and mobile. The ear, nose and throat examinations were unremarkable. 
USG with Doppler flow of neck was performed and revealed no focal lesion seen on right neck. Right IJV appeared larger compared to the left IJV at rest (Figure 1). However, enlarged right internal jugular vein noted during Valsalva maneuver (Figure2). Right IJV appeared to have normal vascularity with no evidence of thrombosis within (Figure 3). Early diagnosis of IJV ectasia was established and the child was treated conservatively with regular serial follow up and ultrasound of neck biannually.

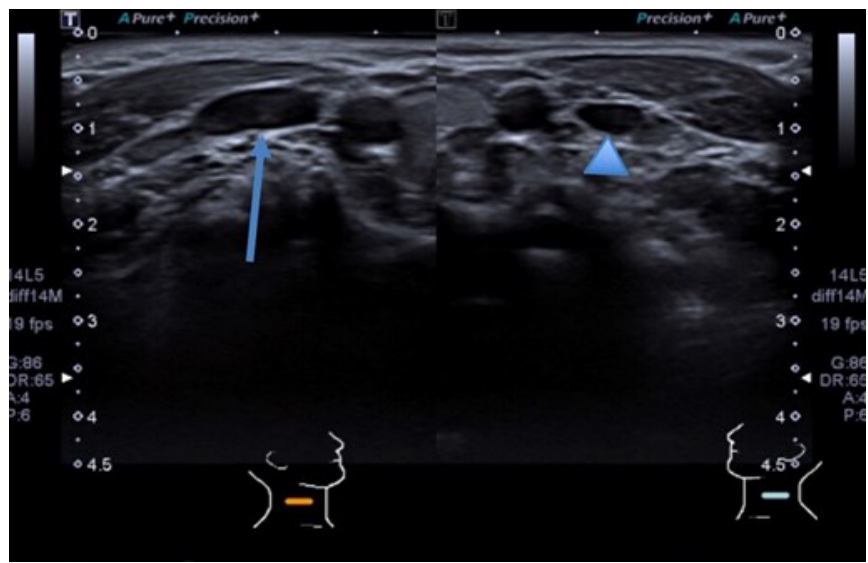

Figure 1 : Comparison of right (arrows) and left IJV (arrowheads) on rest. Right IJV appeared to be larger.

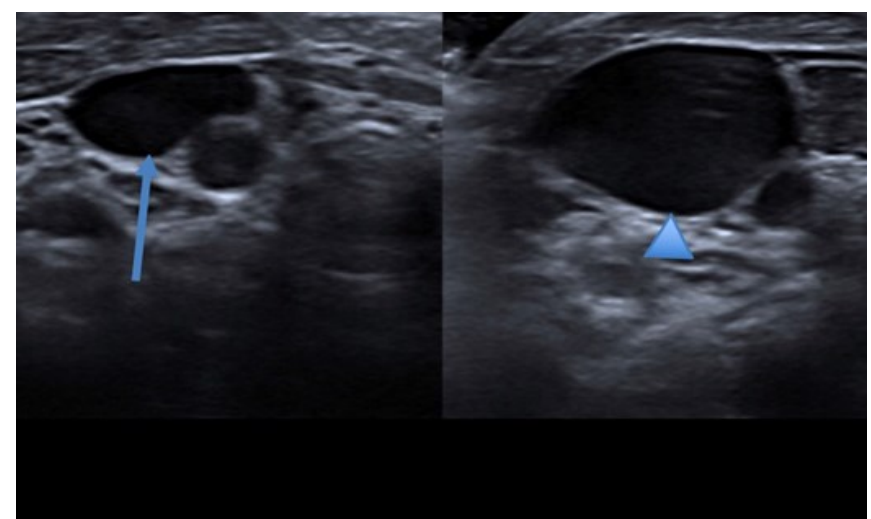

Figure 2: Comparison of right IJV on rest (arrows) and upon vasalva (arrowheads). Marked increase in size of IJV during vasalva.

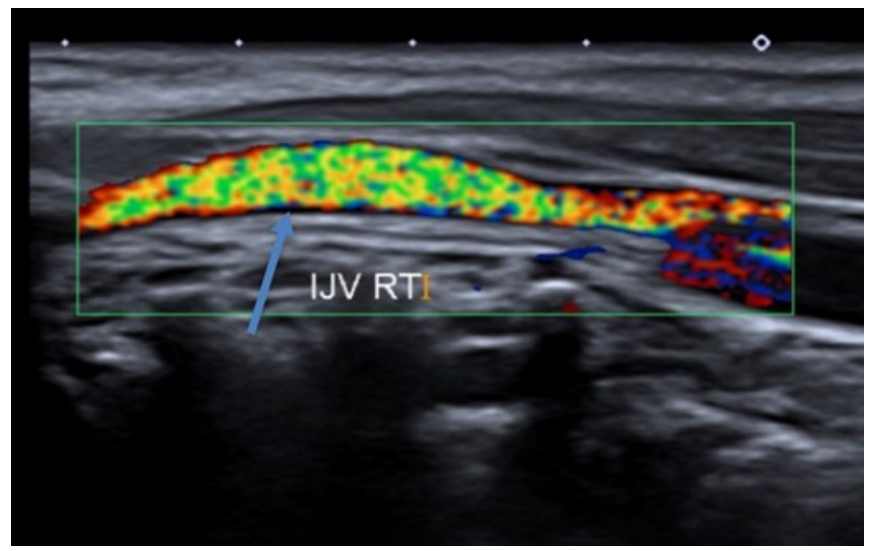

Figure 3: Doppler USG revealed presence of venous blood flow in right IJV (arrows) without thrombosis within

\section{DISCUSSION}

Venous ectasia is rare. It has numerous terminologies such as venous aneurysm, venous pseudoaneurysm, congenital venous cyst, phlebectasia, venous cyst and venoma. ${ }^{4}$ The disease favors male children and involved the right Internal Jugular Vein (IJV) 4 times more common than the left side. ${ }^{5}$ Regardless of various theories of etiology that have been postulated over the past decades, the cause of jugular ectasia remains controversial. Many authors proposed the hypothesis of jugular ectasia is due to congenital structural defect in the vein wall, mechanical pressure, acquired lesion of the vein, gross anatomic abnormality and idiopathic causes. ${ }^{6}$ One of the significant hypothesis favors of right IJV ectasia proposed by Anderhuber et al is high pressure of retrograde flow in the right IJV. ${ }^{7}$ The retrograde flow is from short, less angulated and valveless right brachiocephalic vein (Figure 4).

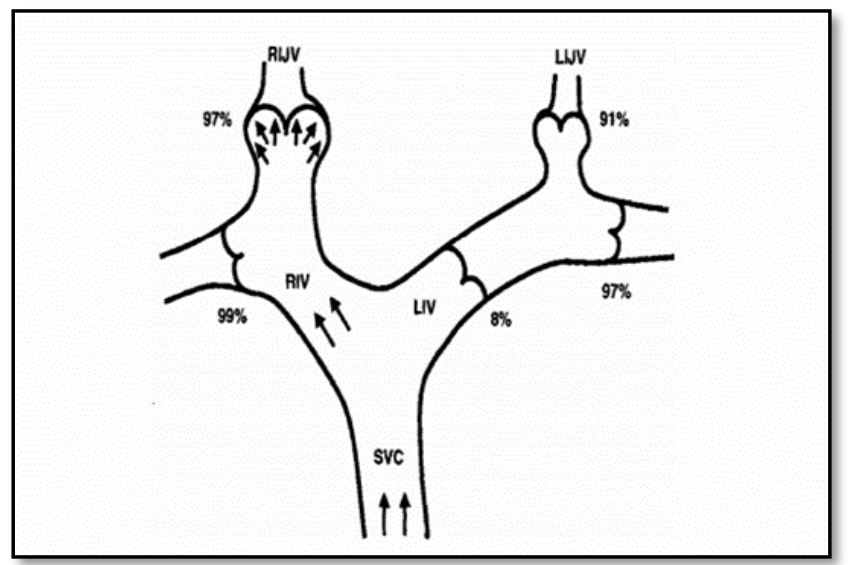

Figure 4: Proposed mechanism for preferential dilatation of the right jugular bulb. The percentage indicate the presence of competent valves. Shorter, less angulation and valveless of Right brachiocephalic vein favors for Right IJV ectasia.

Venous ectasia commonly presented with intermittent prominent swelling associated with an increase in intrathoracic pressure. It may contribute to compressible symptoms such as dysphonia, dysphagia and difficulty in breathing. ${ }^{8}$ Unfortunately, most patients are asymptomatic leading to a diagnostic dilemma. Some differential diagnosis of non-pulsatile soft neck masses which becomes prominent on increased intrathoracic pressure may include laryngocele or external laryngeal diverticulum, cupular inflation and tumors or cysts of the upper mediastinum. ${ }^{9}$

The typical clinical presentation of jugular ectasia with additional Doppler USG is almost 
pathognomonic. USG with Doppler flow is proven to be a gold standard to diagnose jugular ectasia. ${ }^{3}$ USG is a short procedure and non-invasive technique. USG is capable to differentiate between a cystic and solid mass while Doppler flow imaging able to recognize the blood flow direction and any thrombosis within the vessel. Valsalva maneuver during USG will show an increase of vessel diameter in IJV ectasia. In our case, the patient's abdomen was palpated to increase the intrathoracic pressure. Contrastenhanced CT scan of the neck region is another mode of imaging modality proven to diagnose this condition as it can define the spatial relationship, differentiating the lesion from other cystic and vascular malformation. However, risk of the contrast, sedation, radiation and cost-effectiveness is in favor of the ultrasound Doppler imaging alone. Magnetic resonance angiogram or venogram is another imaging modality that can cater to diagnose IJV ectasia.

Early diagnosis of IJV ectasia will grants confident reassurance to the parents due to its benign nature. A life-threatening condition such as ruptured ectasia causing a hematoma, compression to the surrounding vital structures and airway compromise are yet to be reported. Despite the known main clinical risk of ectasia are Horner's syndrome ${ }^{2}$ and thrombosis ${ }^{3}$, the incidence of such complications are still unheard.

Most authors recommend conservative follow up for this benign self-limiting condition unless the patient develops complications as mentioned before. Serial follows up and biannually Doppler USG monitoring is recommended to look for progression, size and thrombosis risk. ${ }^{3,4}$ Cosmetic reasons and complications of IJV ectasia may require ligation of the feeding vessels followed by surgical resection of the dilated segment. ${ }^{10}$

\section{CONCLUSION}

Jugular ectasia should be included in the differentials of a benign neck mass in children despite infrequently encountered. Dilated jugular vein on ultrasound Doppler on Valsalva maneuver is pathognomic of jugular ectasia. Early diagnosis with serial follow up can reduce parent's anxiety and will reduce complications.

\section{REFERENCES}

1. Abdulla AS, Aldabagh MH. Congenital phlebectasia of the internal jugular vein (case report). Duhok Medical Journal. 2008;2(1):15560.

2. Inci S, Bertan V, Kansu T, Cila A. Horner's syndrome due to jugular venous ectasia. Child's Nervous System. 1995 Sep 1;11(9):533-5.

3. Rao KN, Chavan SS, Kale VD, Hekare A, Sylendran A, Khond A. IJV Phlebectasia: an approach algorithm. International Surgery Journal. 2017 Sep 27;4(10):3570-2.

4. Bowdler DA, Singh SD. Internal jugular phlebectasia. International journal of pediatric otorhinolaryngology. 1986 Dec 1;12(2):165-71.

5. Al-Dousary $\mathrm{S}$. Internal jugular phlebectasia. International journal of pediatric otorhinolaryngology. 1997 Jan 3;38(3):273-80.

6. Danis RK. Isolated aneurysm of the internal jugular vein: a report of three cases. Journal of pediatric surgery. $1982 \mathrm{Apr}$ 1;17(2):130-1.

7. Anderhuber $F$. Venous valves in the large branches of the vena-cava superior. Acta Anatomica. 1984 Jan 1;119(3):184-92.

8. Balik E, Erdener A, Taneli C, Mevsim A, Sayan A, Yüce $G$. Jugular phlebectasia in children. European journal of pediatric surgery. 1993 Feb;3(01):46-7.

9. Zohar Y, Ben-Tovim R, Talmi YP. Phlebectasia of the jugular system. Journal of CranioMaxillofacial Surgery. 1989 Feb 1;17(2):96-8.

10. Kumar GV, Reddy BR, Bhat SM, Vuppala R. An intriguing case of ectasia of external jugular vein presenting as intermittent neck mass. Indian Journal of Otolaryngology and Head \& Neck Surgery. 2016 Sep 1;68(3):367-9. 